\title{
Food and Mood: Just-in-Time Support for Emotional Eating
}

\author{
Erin A. Carroll \\ University of Rochester, Computer Science \\ Rochester, NY 14627 USA \\ erinc@cs.rochester.edu
}

\author{
Mary Czerwinski, Asta Roseway, \\ Ashish Kapoor, Paul Johns, Kael Rowan \\ Microsoft Research \\ Redmond, WA 98052 \\ $\{$ marycz, astar, akapoor, paul.johns, \\ kael.rowan\}@microsoft.com
}

\author{
m.c. schraefel \\ University of Southampton \\ Southampton, Hampshire, UK \\ mc+ac@ecs.soton.ac.uk
}

\begin{abstract}
Behavior modification in health is difficult, as habitual behaviors are extremely well-learned, by definition. This research is focused on building a persuasive system for behavior modification around emotional eating. In this paper, we make strides towards building a just-in-time support system for emotional eating in three user studies. The first two studies involved participants using a custom mobile phone application for tracking emotions, food, and receiving interventions. We found lots of individual differences in emotional eating behaviors and that most participants wanted personalized interventions, rather than a pre-determined intervention. Finally, we also designed a novel, wearable sensor system for detecting emotions using a machine learning approach. This system consisted of physiological sensors which were placed into women's brassieres. We tested the sensing system and found positive results for emotion detection in this mobile, wearable system.
\end{abstract}

\section{INTRODUCTION}

We eat not just because we are hungry and craving nutrients but also for a host of emotional and habitual reasons. There is no single term that encompasses the combination of lifestyle, hedonic, emotional, or habitual over-eating that leads to obesity. For this paper, we will use the term "emotional eating" as a placeholder for non-homeostatic eating (i.e., eating that is not physiologically required). Persuasive technologies have been used within the health space to assist people in developing new habits around health, from making better eating choices to encouraging a more active lifestyle. These systems have, for the most part, relied on fixed contexts to offer support: alarms at particular times of the day; showing performance updates when one glances at their mobile phones [1]; and user-activated support $[2,3]$. However, one cannot rely solely upon fixed solutions for persuasive technology to effect longterm behavioral change. An alternative approach is to intervene proactively to prevent the behavior from happening. In the case of emotional eating, our goal is to provide an intervention before the person turns to food for emotional support.

We begin to investigate just-in-time interventions to support behavior modification for emotional eating. Designing such a system is an ambitious endeavor: it involves exploring the emotional triggers of eating, developing elaborate technology for automatically detecting emotions, and investigating intervention approaches for emotional eating. In this paper, we present three user studies that move us towards the goal of developing a just-in-time support system for emotional eating. The purpose of the first study was to gather data about emotional eating patterns. The second study investigated the feasibility and benefits of emotional eating interventions. Finally, the third study focused on emotion detection with machine learning and custom-built wearable sensors.

\section{RELATED WORK}

\section{A. Persuasive Health Technologies}

Persuasive technologies for health and weight management have become ubiquituous over the past few years with several off-the-shelf fitness technologies like FitBit ${ }^{1}$ and BodyBugg ${ }^{2}$ helping to make the logging of activity and physiological state more accessible. These fitness gadgets are designed to help people measure physical activity and sleep quality and to motivate increased movement. Amft and Troster [4] built sensors for monitoring chewing and swallowing, and they proposed how these sensors could be included in an elaborate, (yet uncreated) persuasive system for health. Purpura et al. also proposed a similar, uncreative persuasive system [5]. The technology in their system included, to name a few parts: an earpiece for monitoring chewing and swallowing; augmented reality glasses for capturing foods consumed; and heart rate recording for sensing exercise. This proposed system also connected through a mobile phone application in order to process the data and for the person to receive feedback. The hypothetical feedback, whether from a social network, a close friend, or pre-recorded messages, served as a health intervention to encourage the person to be more active or consume less food.

Not at all persuasive systems need to be as elaborate as those discussed above. A persuasive system with peer support alone can help people be more successful in weight loss. Mutsaddi et al. [6] utilized text messages as a form of social support for encouraging more physical activity. They found that even after the novelty of text messages wore off, the text messages were still beneficial because they served as reminders to participants to be more active.

\section{B. Eating for Non-Homeostatic Reasons}

There are many theories around why we eat. One is largely homeostatic: we eat because we need fuel to survive; we crave certain foods because we require certain nutrients to function [7]. Other reasons are more nuanced, though these too are intertwined in physiological responses. For instance, many people reach for calorically dense foods, like donuts, when stressed. Gilhooly et. al characterized these responses as "instinctive" in that at some point these behaviors may have served a survival function [8]. Stress releases hormones, which trigger a fight or flight response; thus, grabbing high-energy, available foods (like the sugary donut) would be effective

\footnotetext{
${ }^{1}$ FitBit: http://www.fitbit.com

${ }^{2}$ BodyBugg: http://www.bodybugg.com
} 
for energy production. Likewise, when confronted with the variety of a buffet, it seems a food scarcity mentality kicks in, and more food is taken in than necessary. The challenge in a food abundant culture is that these instinctive responses are no longer helpful. An important insight around over-eating behavior is that non-homeostatic eating patterns can be reeducated [8]. In other words, both our physiological responses (the release of ghrelin to cue stomach grumbling at particular times [7]) and psychosocial responses (eat in the presence of food or in response to stress) are malleable. Therefore, technology that is used to intervene before the maladaptive behavior happens could provide some assistance towards longterm, behavioral change.

\section{Supporting Behavior Change}

An increasingly well-regarded approach to support eating behavior change is Cognitive Behavioral Therapy (CBT) [9]. The focus of CBT is to help a person become aware of their "maladaptive behaviors" and then replace these with adaptive ones "by modifying their antecedents and consequences and by behavioral practices that result in new learning" [10]. A common approach to supporting discovery of these antecedents to maladaptive behaviors, such as one's cues for emotional eating, is to have participants in CBT do work to identify triggers. Typical approaches include keeping daily food and mood logs or journals. In some cases, the goals of the logs are to highlight particularly positive states ("benefit finding") to enhance success with new, adaptive behaviors [11]. One particularly relevant process has been called "real-time self-monitoring" around eating, related behaviors, and feelings [12]. The rationale for the criticality of monitoring is that "it helps patients to be more aware of what is happening in the moment so that they can begin to make changes to behavior that may have seemed automatic or beyond their control" [12]. An important aspect of CBT is bringing the automatic or thoughtless actions into consciousness for deliberate engagement/change.

\section{Implicit Emotion Detection}

Emotion detection with sensor data has been carried out in the past. McDuff et al. used a variety of signals (i.e., electrodermal activity, posture, facial expressions, etc.) to detect emotions using a machine learning approach with selfreported ratings of emotion serving as the ground truth in their emotion classifiers [13]. They found that the electrodermal activity (EDA) signal was the most beneficial, which is a measure of the eccrine sweat glands [14,15]. McDuffs et al.'s detection system involved sensors that required users to be tethered to their desk (e.g., Kinect, web cam, etc.).

The goal of our system was to perform emotion detection in a mobile, wearable system, which allows us to collect data as users move about their day. Chang et al. created a mobile system for detecting user emotion but this was done via activity modeling and speech prosody tracking [16]. In contrast, the goal of our system was to use on-body sensors with EDA and electrocardiogram (EKG), since these sensors have been shown to be reliable for emotion detection $[14,17,18]$.

\section{RESEARCH APPROACH}

Designing a system to provide just-in-time interventions for emotional eating is an ambitious endeavor. Consider the following hypothetical scenario:
Sally has been home from work for a few hours, and she finds herself rather bored. An application on Sally's mobile phone has also detected that she is bored by reading her physiological state through wearable sensors. Since this mobile application has previously learned that Sally is most susceptible to emotional eating when she is bored, the application provides an intervention to distract Sally and hopefully prevent her from eating at that moment.

From this scenario, we see three key requirements for a just-in-time support system for emotional eating. First, the application has to be aware of the user's emotional eating patterns. Does Sally emotionally eat only when she is bored? Second, the system needs to be able to implicitly detect emotions. This involves wearable physiological sensors that are connected to the mobile phone. Implicit detection of emotions would then be possible through machine learning classification, which requires training on large amounts of users' data. Finally, it is critical (and perhaps the most challenging) to determine how to intervene. What type of intervention do we design? How often do we intervene? How do we prevent it from becoming an annoyance to the user?

Our approach to researching a just-in-time support system for emotional eating was to make strides towards addressing these three requirements. We studied these requirements across three user studies, which have been summarized below.

- Study 1: Gather Emotional Eating Patterns. We investigated eating behaviors and corresponding emotions of participants by having them self-report their emotions and log their eating patterns using a custom built application called EmoTree. The goal was to understand their emotions associated with eating.

- Study 2: Investigate An Intervention Technique. The purpose of Study 2 was to learn about a particular intervention technique for emotional eating. We prototyped implicit intervention by triggering an intervention based on self-reported ratings of emotions. This allowed us to gather early feedback about interventions before implementing an automatic system. Are users aided by the intervention? Was the intervention sent at the appropriate time? What other types of interventions would interest users?

- Study 3: Emotion Detection with Wearables. This work was a first step in building an automatic system. We investigated the feasibility of using physiological sensor data, combined with machine learning, to automatically detect emotions in a mobile system. We also present the design of our wearable system.

\section{EmoTree: Mobile Phone Application}

EmoTree is a custom designed Windows 7 mobile phone application, consisting of four screens. The default screen provides an overview of the user's logging activity and overall sentiment (Figure 1A). The tree on this screen eventually populates its leaves over time, as the user interacts with the app. Each circle represents a day's worth of activity and the color green is used to indicate positive decisions based on food intake. The user's sentiment is aggregated as the background sky color to indicate positive or negative valence of affect. 


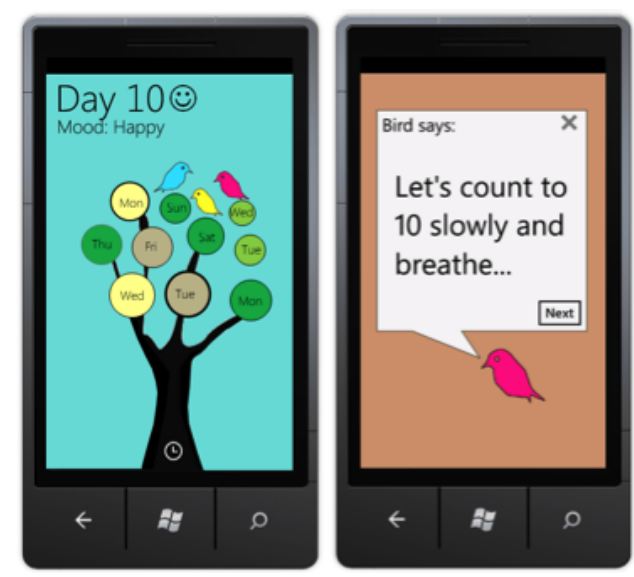

Fig. 1. EmoTree Mobile Phone Application. Main Page (A) and Intervention Page (B)

As usage continues, the user may assess overall progress or history by selecting the history icon at the bottom of the tree. Inspiration for the interface design came from the notion of tending a garden and using that as a metaphor to visually guide users to make better choices [1]. Since the application was designed for long-term use, the idea of a tree that gradually grows based on your daily health decisions served best as a visual guidance system that could invoke encouragement: the healthier your choices, the healthier the tree.

To start using the application, the user goes to the main screen (Figure 1A) and glances at their current mood. By swiping to the left, the user is taken to a screen that asks: "How do you feel?" (Figure 2B) Self-reporting of emotion was based on the Russell's Circumplex model [15], widely used across the affective computing community $[13,19]$, in which emotion is represented two-dimensionally; valence on the $\mathrm{x}$-axis and arousal on the y-axis. Figure 1A shows the emotion self-report tool that participants used. In our user interface, we used the terminology of "Negative" to "Positive" to describe valence, and "Pumped" to "Relaxed" to describe arousal, as in McDuff et. al [13]. After indicating their current emotional state, the users also indicated how engaged they were with their current task, on the same screen. Reminders to self-report emotions were sent to the users' mobile phones every hour, on the hour.

Users were also instructed to swipe right from the main screen to log any food that had been consumed (see Figure 2A) for what they were asked to log. Logging food populated the leaves on the main screen. After logging food, the user was immediately sent to the self-report page to indicate their emotions prior to eating. The task engagement question was not asked after logging food.

Lastly, we introduce the intervention screen (Figure 1B). which consisted of a deep breathing exercise. Users were instructed to tap on the screen for each breath that they took for 10 seconds (or 10 taps). The intervention was employed in Study 2 and will be discussed further in Section VI.

\section{Study 1: Gather Emotional Eating Patterns}

In Study 1, we conducted a user study ( $\mathrm{N}=12,2$ males) to investigate emotional eating patterns using EmoTree. In recruitment, all participants self-identified as emotional eaters.

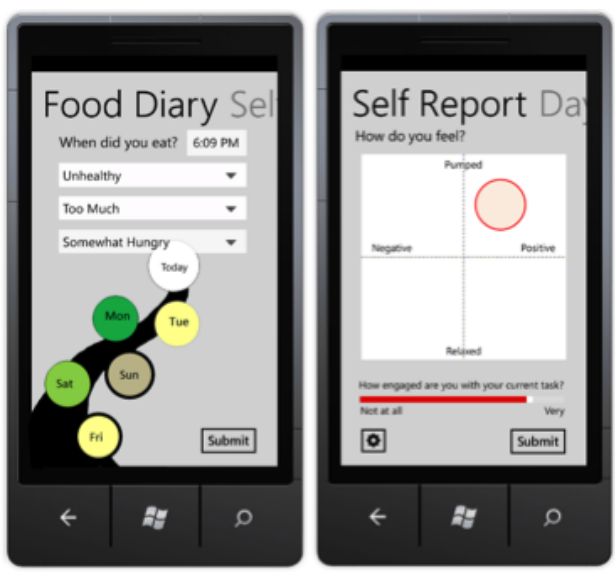

Fig. 2. EmoTree Mobile Phone Application. Nutrition Log (A) and SelfReport Tool (B)

Prior to the study, users filled out a pre-experiment survey that asked about their emotional eating patterns, called the Emotional Eating Scale [20]. All participants were given a demonstration of the EmoTree app, and they were given instructions on how and when to use the software. This study took place over a period of four days (Tues-Fri). Participants were asked to use EmoTree to self-report emotions every hour, on the hour, in addition to reporting food. We collected at least 10 self-reports of emotion per day from participants.

\section{A. Analysis and Results}

Our pre-survey results on the Emotional Eating Scale showed that the emotions marked as causing a moderate to strong urge to eat included: on edge, nervous, upset, worried, nothing to do, bored, irritated, restless, and discouraged. These findings support research on emotional eating as reported elsewhere [21].

From the four days of data collection, we created two scatterplots for each person ( 2 plots x 12 participants). The first scatterplot was of a person's emotional ratings, which visualized their emotional state over the duration of the study. The second scatterplot was of a person's emotional ratings that were associated with food, showing us their emotional state just before eating. It was not surprising to find that there were a lot of individual differences and that people ate when they were experiencing a variety of emotional states. In fact, most users had eating events that occurred in all four quadrants of the Circumplex model (Figure 2B). However, we did find that six participants tended to eat when they were predominantly stressed with the majority of eating events occurring in the upper-left quadrant (i.e., negative \& pumped). We also observed across all participants that few eating events happened when the users were in a calm/serene state, so very few eating events were in the lower-right quadrant (i.e., positive \& relaxed).

\section{B. Intervention Technique}

We decided to intervene based on when users provided selfreports occuring in the upper-left quadrant of the Circumplex model (i.e., negative \& pumped). Our goal was to explore if our intervention could help move participants from a stressful state to a calmer state. Therefore, we developed a deep breathing 
activity (Figure 1B), where a little bird is displayed that counts users' breaths with each tap on the screen. Several components are at work in our design decisions. First, stress has been identified as a contributor to obesity and to emotional eating [7,21]. Second, we found in the analysis of EmoTree's emotional eating data that 6 out of 12 participants were primarily stress eaters, and the Pre-Survey results also show that participants primarily eat in response to feelings that were associated with stress (e.g., on edge, upset, worried). Finally, we leverage a CBT strategy for reducing emotional eating, as described in Section II-C: by targeting stressful moments that may be precursors to an emotional eating episode, the breathing exercise may break the focus on stress, allowing for further cognitive processing of what one may be about to do, thus offering the opportunity to choose a different, and more positive, action.

\section{Study 2: InVESTIGATE InTERVEntion TeChniQues}

In Study 2, we released a new version of EmoTree to participants, which included a deep breathing intervention that was triggered in response to self-reported emotions of stress/anxiety. This study was focused on the usefulness of the deep breathing intervention technique.

This study was comprised of the same participants from Study 1 with the exception of one who withdrew due to travel. This study also took place over a period of four days (TuesFri). During this time, participants $(\mathrm{N}=11,1$ male) continued to use EmoTree for tracking food and self-reporting emotions throughout the day, using the same instructions from Study 1. We investigated intervention effectiveness with Pre- and PostExperiment surveys. The Pre-Survey included questions about participant awareness of eating behaviors and emotions as a result of tracking emotions and nutrition in Study 1. The PostSurvey asked questions about the success of the deep breathing activity (i.e., effectiveness in preventing emotional eating and for reducing stress/anxiety); whether triggering the intervention based on stress/anxiety was effective; and solicited suggestions for alternative intervention techniques. The Post-Survey sought to understand whether we should build a fully automated system, and if so, how a particular intervention should be implemented. Specifically, we asked participants questions about whether they would be interested in the system; how successful the deep breathing activity was (i.e., effectiveness in preventing emotional eating and for reducing stress/anxiety); whether intervening based on stress/anxiety was effective; and suggestions for alternative intervention techniques.

\section{A. Analysis \& Results}

In the Pre-Survey, all participants reported that they became more aware of their eating behaviors, and $87.5 \%$ reported that they became more aware of their emotions. Qualitative feedback also indicated that logging in EmoTree was an excellent awareness tool:

- $\quad$ "I was eating without being aware of it, but by having to log both my eating habits and my emotions, I became aware of triggers for emotional eating, and also more aware of the health (or lack thereof) in my diet."
- "I became more conscious when I was about to eat or drink and self-reflected on why I was consuming something."

- "I noticed more how I was feeling when I ate, and this was not something you normally stop to think about."

However, only $37.5 \%$ reported that their eating behaviors changed as a result of Study 1, so while logging made participants more aware, it seemed that most needed something extra to incent real change.

The Post-Survey was given to participants after completing Study 2. The Post-Survey asked participants how accurate the stress/anxiety self-reporting trigger was for the intervention timing. The average accuracy was $2.80(\mathrm{SD}=1.30)$ on a scale of 1 (Not At All Accurate) to 5 (Extremely Accurate). This result was not surprising given that we knew from Study 1 that our participants ate for a variety of reasons, not just when stressed. We also asked how effective the deep breathing intervention was at reducing stress $(\mathrm{M}=3.0, \mathrm{SD}=1.87)$ and the effectiveness of deep breathing in preventing emotional eating $(\mathrm{M}=2.20$, $\mathrm{SD}=1.30$ ). These last two questions were on a scale of 1 (Not At All Effective) to 5 (Extremely Effective). We were pleased that the deep breathing worked moderately at reducing stress, but its effectiveness at preventing emotional eating was not as strong as we might have hoped for.

Participants were also asked about suggestions for other intervention techniques. We learned that personalization is desirable for intervention, not only in regards to individual differences in eating patterns but also for personal preferences about interventions. For instance, 3 out of 11 participants were enthusiastic about the deep breathing activity and tried to incorporate it throughout their day, but the others wanted alternatives. The alternative interventions that they suggested, included: presenting something funny to alleviate boredom or stress; having them fill out a gratitude questionnaire (i.e., reminding them what they have to be thankful for); meditation; suggestions to take a walk; brain teasers/games; asking if they are really hungry; reading or writing; and calling a friend. From these results, personalizing the intervention would be the best solution. However, offering a menu of these interventions could break the food focus and prove to be a useful approach, which is consonant with intervention work in CBT.

\section{Study 3: EMotion Detection With WeARABles}

In Study 1 and 2, we explored emotional eating patterns and investigated the feasibility and benefits of developing an elaborate, integrated system, as described in the scenario. We found some initial results that suggested providing awareness and just-in-time support for emotional eating could work with better personalization on timing and intervention. To move towards the goal of a personalized, integrated system, this study was focused on investigating the feasibility of using physiological sensors to implicitly detect emotions. While implicit emotion detection has been done in the past [13], this is the first study, that we are aware of, that makes use of wearable, mobile sensors for detecting emotions. In this section, we discuss our novel sensing system, including the sensors employed and the wearables. We also discuss our methodology for collecting data. Finally, we present positive results for classifying emotions using wearable sensors. 


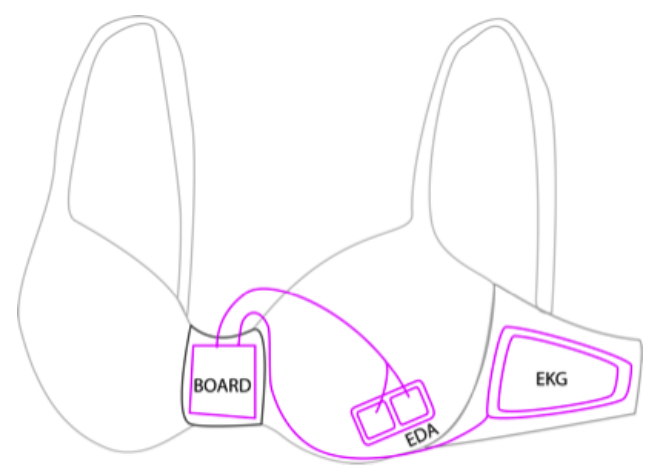

Fig. 3. Sensor placement outline, showing where EKG and EDA was collected and connected to the custom board.

\section{A. Emotion Sensing System}

1) Sensors: We used custom boards called GRASP (Generic Remote Access Sensing Platform) which is a realtime system and is comprised of a physical sensor board, corresponding firmware, software libraries, and an API. The GRASP board has an MSP-430 microprocessor and is powered by a Lithium-Ion polymer (3.7V) battery. GRASP can sample up to eight bio-signal channels (TI80S1298) simultaneously. The GRASP boards in this study were configured to capture heart rate and respiration with an electrocardiogram (EKG) sensor; skin conductance with an electrodermal activity (EDA) sensor; and movement with a 3-axis accelerometer and a 2axis gyroscope. The board transmitted the sensor stream data to a mobile phone application using Bluetooth, which was then stored remotely into a Microsoft Azure Cloud.

2) Design of Wearables: The design motivation for the bra sensing system (Figure 3) was driven by a few key factors. First, we needed a form factor that would be comfortable when worn for long durations. We also needed a way to gather both EKG and EDA signals; so ideally, we wanted to collect those signals from the same wearable. The bra form-factor was ideal because it allowed us to collect EKG near the heart. Ultimately, we chose to leverage the functionality and wearability of a bra, but had to consider that each participant's chest and rib size would vary greatly. Rather than build each participant their own embedded sensor bra, we aimed for a much more modest solution: conductive pads that could be inserted or removed. This provided us flexibility in recruiting participants, in addition to resolving the wash-ability aspect. Three pads for each participant were required in order to capture both EKG and EDA (Figure 3). For EKG, two pads were designed to fit snug against each side of the ribs. These pads were designed with more surface space to help reduce noise in the signal. The EDA pad was initially designed to fit on the backside of the bra; however, we were unable to gather sufficient signal due to low sweat levels in the back. We therefore had participants relocate the EDA pad into the bra cup, just under the breast. It is worth nothing that collecting EDA from this part of the body is non-standard; however, EDA was still a useful signal.

The board sits at the center of the user's sternum (Figure 3), encased in a fabric pouch that was stitched to the outside of the bra. Each sensor pad is comprised of $2.5 \mathrm{~mm}$ of laser cut neoprene sandwiched with batting and laser cut conductive silver ripstop fabric (Figure 4). A laser cut outline of cotton was top stitched by a sewing machine to enclose all the

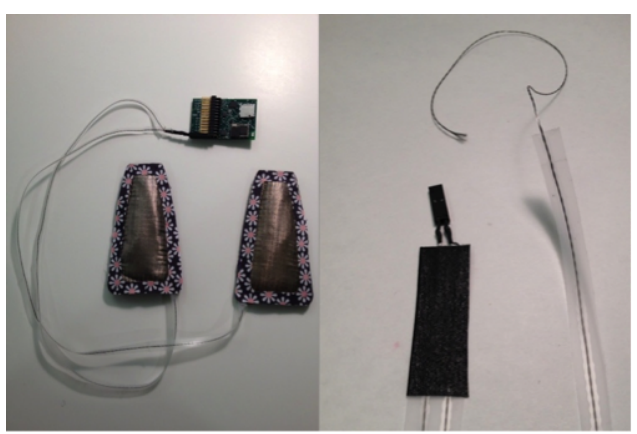

Fig. 4. EKG sensor pads, connected to a GRASP board (A); Conductive thread insulated by scotch tape (B)

material. The insulated wire turned out to be inflexible and obstructive to the wear, so we explored ways in which we could make an electrical connection using conductive thread. For insulation, we sandwiched the thread with two pieces of scotch tape. This method worked remarkably well in that it retained the soft and flexible nature of the thread but was also not noticed when worn. One end of the conductive thread was sewn into the pad and then covered by a top stitch, while the other end was tied to thin wire, heat wrapped, and crimped to a connector on the GRASP board. The average conductivity between the sensor and connector was approximately $12 \mathrm{ohms}$.

3) Methodology: We conducted a user study to see if emotion detection through physiological sensing was possible in a mobile system. To this end, we had four women from our research lab participate in this study. The study involved these women wearing the three conductive fabric pads inside their bras, as previously described. They also used the mobile application, EmoTree, in order to self-report their emotions, which was used as labeled data in our machine learning classifier (ground truth).

Participants wore the bra sensing system and reported their emotions for about 4-6 hours a day over a period of approximately four days. It was very tedious for participants to wear our prototyped sensing system, as the boards had to be recharged every 3-4 hours, which resulted in participants having to finagle with their wardrobe throughout the day.

4) Analysis and Results: Similar to McDuff et. al [13], we took a machine learning approach for predicting emotions. The sensor data went through several pre-processing stages before we could perform classification. First, we applied two different filters to the raw EKG signal: a $2 \mathrm{~Hz}$ low-pass filter to get respiration and a $2 \mathrm{~Hz}$ high-pass filter to get heart rate. Next, we normalized all 8 signals (heart rate, respiration, EDA, 3axis accelerometer, 2-axis gyroscope) between 0-1. Finally, we extracted features from the signal by creating 5 bands that fell within our normalized values. These bands were: 0 to $<0.2$, 0.2 to $<0.4,0.4$ to $<0.6,0.6$ to $<0.8$, and $>0.8$. We used these bands to determine within a 10 -minute time period the proportion of data that occurred within each band. This feature extraction process resulted in 40 features ( 8 signals $\mathrm{x} 5$ bands) for each person. The labeled data for classification was selfreported ratings of emotion (x-valence and y-arousal), and the attributes were the 40 extracted features.

We used a classification framework based on Gaussian Process Regression (GPR) [22], which has been shown to 
be very effective for multimodal affect recognition [23] and fits well into the context of our classification task. In particular, we define a similarity (a kernel) function between two observations $x_{i}$ and $x_{j}$ using a Radial Basis Function: $k\left(x_{i}, x_{j}\right)=\exp \left\|x_{i}-x_{j}\right\|^{2} / 2 \alpha^{2}$. GPR considers this similarity when classifying points and assigns labels to test cases such that similar labels are assigned to similar points. We refer readers to Kapoor et. al [23] for further details on affect classification using Gaussian Processes. For testing the classifiers, we used a leave-one-example-out methodology. Specifically, we consider data recorded from all but one data point as the training corpus and then tested the algorithm on the left-out observation. This process was repeated for all points.

We were able to classify arousal at $75.00 \%$ and valence at $72.62 \%$ accuracy. We observe that for both arousal and valence the recognition accuracy is significantly better than chance. Furthermore, we would like to highlight that the recognition accuracy achieved here is at par with other affect recognition systems [13]. Based on these results, we conclude that building a wearable, physiological system is feasible. However, we will continue to explore how to build a robust, real-world system that stands up to every day challenges with regards to battery life, comfortability, and being suitable for both men and women. Since these classification results were based on log files, rather than real-time sensor data, our next iteration will also run using real-time sensor data that is able to predict emotions and show an appropriately time, personalized intervention.

\section{CONTRIBUTIONS AND FUTURE WORK}

In this paper, we began to investigate just-in-time intervention to support behavioral modification in emotional eating. Designing a just-in-time support system for emotional eating is an ambitious endeavor, and to this end, we presented three user studies that moved us towards the goal of developing a fully integrated system. We found in Study 1 from user logging that 6 out of 12 participants primarily ate when they were stressed. In Study 2, we found that participants were enthusiastic about interventions for emotional eating, but that most wanted personalized interventions, rather than the single, pre-determined deep breathing interventions. Finally, in Study 3, we investigated using wearable sensors to implicitly detect emotions while participants were mobile. Using log files, we were able to detect arousal at $75.00 \%$ and valence at $72.62 \%$.

There are several future research directions that are essential to building an integrated system for just-in-time support. First, we have already expanded our implicit emotion detection system to work using real-time sensor data, opposed to log files. While the wearable sensors in the brassiere form factor only allowed women to participate in Study 3, we have now moved towards using the Affectiva $\mathrm{Q}^{3}$ sensor bracelets for this collection. Our pilot results have been quite promising for men and women. Finally, we are currently exploring the just-intime intervention space in which we test different intervention approaches in longitudinal studies. We consider research in intervention design to be critical in future iterations, including personalized methods of responding to the user, in addition to investigating formal theories of behavior change [24].
${ }^{3}$ Affectiva Q: http://www.affectiva.com

\section{REFERENCES}

[1] S. Consolvo, P. Klasnja, D. W. McDonalds, D. n. Avrahami, J. Froehlich, R. Libby, K. Mosher, and J. A. Landay, "Flowers or a robot army? Encouraging awareness \& activity with personal, mobile displays," in ACM UbiComp 2008, pp. 54-63, 2008.

[2] P. Y. Chi, J. H. Chen, and J. L. Lo, "Enabling Calorie-Aware Cooking in a Smart Kitchen," Persuasive 2008, pp. 116-127, 2008.

[3] D. Mankoff, G. Hsieh, H. C. Hung, S. Lee, and E. Nitao, "Using LowCost Sensing to Support Nutritional Awareness," in Proceedings of ACM UbiComp 2002, pp. 371-387, 2002.

[4] O. Amft and G. Troster, "On-body sensing solutions for Automatic Dietary Monitoring," IEEE Pervasive Computing, vol. 8, no. 2, pp. 6270, 2009.

[5] S. Purpura, V. Schwanda, K. Williams, W. Stubler, and P. Sengers, "Fit4Life: The design of persuasive technology promoting healty behavior and ideal weight," in Proceedings of ACM CHI 2011, pp. 423-434, 2011.

[6] A. Mutsaddi and K. Connelly, "Text Messages for Encouraging Physical Activity: Are they effective after the novelty effect wears off?", in Proceedings of Pervasive Health, 2012.

[7] J. F. Davis, D. L. Choi, D. J. Clegg, and S. C. Benoit, "Signaling through the ghrelin receptor modulates hippocampal function and metal behavior in mice," Physiology \& Behavior, vol. 103, no. 1, pp. 39-43, 2008.

[8] C. H. Gilhooly, S. K. Das, J. K. Golden, M. A. McCrory, G. E. Dallal, E. Saltzman, F. M. Kramer, and S. B. Roberts, "Food cravings and Energy Regulation: The characteristics of craved foods and their relationship with eating behaviors and weight change during 6 months of dietary energy restriction," International Journal of Obesity, vol. 31, no. 12, pp. 1849-1859, 2007.

[9] S. G. Hoffman, A. T. Sawyer, and A. Fang, "The empirical status of the "new wave" of CBT," Psychiatr Clin North Am, vol. 33, no. 3, pp. 701-710, 2010.

[10] C. Otte, "Cognitive behavioral therapy in anxiety disorders: Current state of the evidence," Dialogues in Clinical Neuroscience, vol. 13, no. 3, pp. 413-421, 2011 .

[11] A. J. Guastella and M. R. Dadds, "Cognitive-behavioural emotion writing tasks: A controlled trial of multiple processes," Journal of Behavioural Therapy and Experimental Psychicatry, vol. 39, no. 4, pp. 558-566, 2008.

[12] R. Murphy, S. Straebler, Z. Cooper, and C. G. Fairburn, "Cognitive behavioral therapy for eating disorders," Psychiatr Clin North Am, vol. 33, no. 3, pp. 611-627, 2010.

[13] D. McDuff, A. Karlson, A. Kapoor, A. Roseway, and M. Cszerwinski, "AffectAura: An Intelligent System for Emotional Memory," in Proceedings of ACM CHI 2012, pp. 849-858, 2012.

[14] W. Boucsein, Electrodermal Activity. New York: Springer-Verlag, 1992.

[15] J. A. Russell, "A circumplex model of affect," Journal of Personality and Social Psychology, vol. 39, no. 6, pp. 1161-1178, 1980.

[16] K. Chang, D. H. Fisher, and J. Canny, "From Affect to Mental Health: A Speech Analysis Library for Unobtrusive Monitoring on Mobile Phones," in 2nd International Workshop on Sensing Applications on Mobile Phones (PhoneSense), 2011.

[17] R. L. Mandryk and M. S. Atkins, "A Fuzzy Physiological Approach for Continuously Modeling Emotion During Interaction with Play Environments," International Journal of Human-Computer Interaction, vol. 6, no. 4, pp. 329-347, 2007.

[18] R. W. Picard, Affective computing. Cambridge, MA, USA: MIT Press, 1997.

[19] C. Latulipe, E. A. Carroll, and D. Lottridge, "Love, Hate, Arousal, and Engagement: Exploring Audience Responses to Performing Arts," in Proceedings of ACM CHI 2011, pp. 1845-1854, ACM Press, May 2011.

[20] A. M. Koball, M. R. Meers, A. Storfer-Isser, S. E. Domoff, and D. R Musher-Eizenman, "Eating When Bored: Revision of the Emotional Eating Scale With a Focus on Boredom," Health Psychology, vol. 31, no. 4, pp. 521-524, 2012.

[21] D. J. Wallis and D. M. Hetherington, "Stress and eating: The effects of ego-threat and cognitive demand on food intake in restrained and emotional stress," Appetite, vol. 43, no. 1, pp. 39-46, 2004.

[22] C. E. Rasmussen and C. Williams, GPML: Gaussian Processes for Machine Learning. Cambridge, MA: MIT Press, 2006.

[23] A. Kapoor, W. Burleson, and R. W. Picard, "Automatic Predictions of Frustration," International Journal of Human-Computer Interaction, vol. 65, no. 8, pp. 724-736, 2007.

[24] E. B. Hekler, P. Klasnja, J. E. Froehlich, and M. P. Buman, "Mind the theoretical gap: interpreting, using, and developing behavioral theory in HCI research," in Proceedings of ACM CHI '13, pp. 3307-3316, ACM Request Permissions, 2013. 